\title{
Mineralização do carbono orgânico em solos tratados com lodo de curtume
}

\author{
Alexandre Martin Martines ${ }^{(1)}$, Cristiano Alberto de Andrade ${ }^{(2)}$ e Elke Jurandy Bran Nogueira Cardoso ${ }^{(1)}$
}

\begin{abstract}
(1)Escola Superior de Agricultura Luiz de Queiroz, Caixa Postal 9, CEP 13418-900 Piracicaba, SP. E-mail: martines@esalq.usp.br, ejbncard@esalq.usp.br (2)Instituto Agronômico, Centro de Solos e Recursos Ambientais, Caixa Postal 28, CEP 13020-902 Campinas, SP. E-mail: andrade@iac.sp.gov.br
\end{abstract}

\begin{abstract}
Resumo - O objetivo deste trabalho foi avaliar a mineralização do carbono orgânico do lodo de curtume, aplicado em solos com diferentes classes texturais, de modo a caracterizar a cinética do processo de mineralização e auxiliar na elaboração de normas técnicas sobre o uso agrícola desse material. Foram conduzidos experimentos de incubação por 105 dias, em laboratório, utilizando-se três solos: Nitossolo Vermelho eutroférrico típico (NVef), Latossolo Vermelho-Amarelo distrófico típico (LVAd) e Neossolo Quartzarênico órtico típico (RQo). O lodo de curtume utilizado nos experimentos foi composto de uma mistura, na proporção de 1:1, do lodo do caleiro e do lodo primário da estação de tratamento de efluentes, resultante da precipitação dos efluentes gerados no processo, com exceção dos efluentes que contêm cromo. As doses aplicadas (base seca) no NVef e LVAd foram equivalentes a 0, 6, 12, 24 e $36 \mathrm{Mg} \mathrm{ha}^{-1}$ e a 0, 3, 6, 12 e $24 \mathrm{Mg} \mathrm{ha}^{-1}$ no RQo. Não se observa prejuízo à atividade microbiana dos solos, avaliada por meio da respiração basal, e a mineralização do carbono adicionado por meio do lodo de curtume é intensa nos primeiros 15 a 20 dias de incubação.
\end{abstract}

Termos para indexação: resíduo orgânico, respiração basal, atividade microbiana.

\section{Mineralization of the organic carbon in soils treated with tannery sludges}

\begin{abstract}
The objective of this work was to evaluate the mineralization of the organic carbon derived from the tannery sludge applied in increasing doses on soils with different textures, to evaluate the decomposition kinetics, as well as to learn about the impact of this residue on the soil microbial community. This knowledge will be helpful for the establishment of technical rules about the agricultural use of the tannery sludges. Incubation experiments were carried out in the laboratory for 105 days, using three soils: a Kandiudalfic Eutrudox (NVef), a Typic Hapludox (LVAd) and a Typic Quartzipsamment (RQo). Tannery sludge used in these experiments was composed of a mixture of liming sludge and the primary sludge from the wastewater treatment station, in the proportion 1:1, with the exception of the wastewater with chromium. The doses of sludge (dry basis) added to the NVef and the LVAd soils were equivalent to 0, 6, 12, 24 and $36 \mathrm{Mg} \mathrm{ha}^{-1}$, and 0, 3, 6, 12 and $24 \mathrm{Mg} \mathrm{ha}^{-1}$ were added to the RQo soil. Soil microbial activity was not inhibited, even by the highest doses, and the most intense carbon degradation occurred on the first 15 to 20 days of incubation.
\end{abstract}

Index terms: organic waste, basal respiration, microbial activity.

\section{Introdução}

O Brasil possui um dos maiores rebanhos comerciais de bovinos do mundo desde 2003, com 195,5 milhões de animais (IBGE, 2003). Segundo Luz (2003), o mercado aparente de peles para curtimento e acabamento está em expansão, com a comercialização de cerca de 35 milhões de unidades por ano.

Cada pele processada gera, em média, $12 \mathrm{~kg}$ de lodo (Claas \& Maia, 1994), sendo o lodo do caleiro e o lodo primário da estação de tratamento de efluentes (ETE) gerados em maior quantidade. Os lodos de curtume são constituídos de materiais orgânicos de origem animal misturados com sais inorgânicos, e alguns desses componentes são nutrientes (nitrogênio, cálcio, enxofre, fósforo, magnésio e potássio) para plantas e microrganismos (Selbach et al., 1991). Esses resíduos se tornam altamente poluidores à medida que concentram elevada carga orgânica e inorgânica, como fenóis, sulfetos, sódio e cromo.

O uso agrícola de lodos de curtume pode contribuir para a melhoria da fertilidade dos solos e nutrição das 
plantas (Konrad \& Castilhos, 2002; Ferreira et al., 2003), além de representar uma forma de descarte do resíduo no ambiente. Quanto a esse último aspecto, deve-se destacar a necessidade de critérios técnicos para aplicação desse tipo de resíduo na agricultura, uma vez que tal prática, quando utilizada de forma inadequada, pode conduzir a elevados valores de $\mathrm{pH}$ (Selbach et al., 1991), sais solúveis (Aquino Neto \& Camargo, 2000; Konrad \& Castilhos, 2002) e cromo nos solos tratados (Figliolia et al., 1992; Aquino Neto \& Camargo, 2000), o que pode comprometer a sustentabilidade agrícola e o uso futuro dessas áreas.

O uso de parâmetros microbiológicos como indicadores da poluição do solo tem sido recomendado, em virtude da maior sensibilidade em detectar alterações de origens naturais e antropogênicas do solo. Segundo Domsch et al. (1983), é importante considerar os efeitos do estresse ao qual as comunidades microbianas são naturalmente submetidas, incluindo as flutuações na temperatura, os extremos de potencial hídrico, extremos de $\mathrm{pH}$, distúrbios físicos do solo, mudanças nas trocas gasosas, decréscimo no suprimento de nutrientes e a presença de predadores e antagonistas. Qualquer alteração em um ou vários desses fatores pode afetar a microbiota.

Dessa forma, o estudo da mineralização da carga orgânica de resíduos, depois da aplicação no solo, pode nortear a definição da dose a ser aplicada a campo, que não prejudique a atividade microbiana no solo.

A medição do carbono liberado pela atividade microbiana do solo, denominada de respirometria, tem sido empregada na avaliação da mineralização dos compostos orgânicos (Brookes, 1995). Alguns dados evidenciam que a fração de mineralização do carbono orgânico aplicado por meio do lodo de curtume, a solos diferentes, pode variar em decorrência da população microbiana mais ativa e diversa de cada solo (BarajasAceves \& Dendooven, 2001).

O objetivo deste trabalho foi avaliar a mineralização do carbono orgânico do lodo de curtume, aplicado em solos com diferentes classes texturais, de modo a caracterizar a cinética do processo de mineralização, auxiliando, desse modo, na elaboração de normas técnicas sobre o uso agrícola desse material.

\section{Material e Métodos}

Foram utilizadas amostras $(0-20 \mathrm{~cm}$ de profundidade) de três solos com diferentes classes texturais:
Nitossolo Vermelho eutroférrico típico (NVef), com textura muito argilosa; Latossolo Vermelho Amarelo distrófico típico (LVAd), com textura argilosa; e Neossolo Quartzarênico órtico típico (RQo), com textura arenosa.

As amostras dos solos coletados foram secadas ao ar, peneiradas (malha de $2 \mathrm{~mm}$ ), homogeneizadas e submetidas a análises para determinação do $\mathrm{pH}$ em água, carbono orgânico, $\mathrm{H}+\mathrm{Al}, \mathrm{S}_{-} \mathrm{SO}_{4}$ extraído com solução de $\mathrm{Ca}\left(\mathrm{H}_{2} \mathrm{PO}_{4}\right)_{2}$, $\mathrm{Cu}, \mathrm{Zn}, \mathrm{Mn}$, e Fe extraído com DTPA, conforme Raij et al. (2001) e P, Ca, Mg, Na, K e Al tocáveis, conforme Silva (1999). A determinação granulométrica das frações areia, silte e argila foi realizada por meio do método da pipeta (Camargo et al., 1986) (Tabela 1).

O lodo de curtume utilizado nos experimentos foi composto de uma mistura na proporção de 1:1 do lodo do caleiro, gerado na etapa de caleiro, e do lodo primário da ETE, resultante da precipitação dos efluentes gerados no processo, com exceção dos efluentes que contêm cromo. Esses lodos foram coletados no Curtume Vanzella, localizado no Município de Rolândia, PR. O lodo de curtume foi caracterizado segundo Kiehl (1985), e o cromo total, determinado segundo Abreu et al. (2001). Os resultados, expressos em matéria seca a $65^{\circ} \mathrm{C}$, estão descritos na Tabela 2.

As doses de lodo utilizadas no NVef (muito argiloso) e no LVAd (argiloso) foram equivalentes a 0, 6, 12, 24 e $36 \mathrm{Mg} \mathrm{ha}^{-1}$ (base seca), considerando $20 \mathrm{~cm}$ de profundidade e densidade do solo igual a $1 \mathrm{~g} \mathrm{~cm}^{-3}$. No RQo (are-

Tabela 1. Caracterização química e granulométrica dos solos estudados.

\begin{tabular}{|c|c|c|c|}
\hline Variável & NVef & LVAd & RQo \\
\hline $\mathrm{pH}\left(\mathrm{H}_{2} \mathrm{O}\right)(1: 2,5)$ & 5,3 & 4,3 & 5,4 \\
\hline Carbono orgânico $\left(\mathrm{g} \mathrm{kg}^{-1}\right)$ & 20,8 & 36,2 & 4,6 \\
\hline $\mathrm{S}-\mathrm{SO}_{4}\left(\mathrm{mg} \mathrm{kg}^{-1}\right)$ & 26,8 & 19,8 & 2,5 \\
\hline $\mathrm{P}\left(\right.$ Mehlich I) $\left(\mathrm{mg} \mathrm{kg}^{-1}\right)$ & 10,0 & 3,4 & 3,1 \\
\hline $\mathrm{K}\left(\mathrm{mmol}_{\mathrm{c}} \mathrm{kg}^{-1}\right)$ & 17,0 & 2,5 & 1,3 \\
\hline $\mathrm{Na}\left(\mathrm{mmol}_{\mathrm{c}} \mathrm{kg}^{-1}\right)$ & 4,7 & 0,6 & 0,0 \\
\hline $\mathrm{Ca}\left(\mathrm{mmol}_{\mathrm{c}} \mathrm{kg}^{-1}\right)$ & 52,7 & 1,7 & 6,2 \\
\hline $\mathrm{Mg}\left(\mathrm{mmol}_{\mathrm{c}} \mathrm{kg}^{-1}\right)$ & 19,0 & 1,5 & 2,8 \\
\hline $\mathrm{Al}\left(\mathrm{mmol}_{\mathrm{c}} \mathrm{kg}^{-1}\right)$ & 2,0 & 18,0 & 3,7 \\
\hline $\mathrm{H}+\mathrm{Al}\left(\mathrm{mmol}_{\mathrm{c}} \mathrm{kg}^{-1}\right)$ & 72,0 & 114,4 & 19,5 \\
\hline $\mathrm{Cu}$ (DTPA) $\left(\mathrm{mg} \mathrm{kg}^{-1}\right)$ & 16,6 & 1,1 & 1,1 \\
\hline $\mathrm{Zn}$ (DTPA) (mg kg-1 $)$ & 4,0 & 0,5 & 0,6 \\
\hline $\operatorname{Mn}(\mathrm{DTPA})\left(\mathrm{mg} \mathrm{kg}^{-1}\right)$ & 244,0 & 1,2 & 7,4 \\
\hline $\mathrm{Fe}(\mathrm{DTPA})\left(\mathrm{mg} \mathrm{kg}^{-1}\right)$ & 23,2 & 282,0 & 18,3 \\
\hline $\mathrm{SB}\left(\mathrm{mmol}_{\mathrm{c}} \mathrm{kg}^{-1}\right)$ & 93,4 & 6,3 & 10,3 \\
\hline $\mathrm{CTC}\left(\mathrm{mmol}_{\mathrm{c}} \mathrm{kg}^{-1}\right)$ & 165,4 & 120,7 & 29,8 \\
\hline Saturação por bases $(\%)$ & 56,5 & 5,2 & 34,5 \\
\hline Argila (\%) & 73,0 & 56,0 & 10,0 \\
\hline Silte $(\%)$ & 23,0 & 4,0 & 2,0 \\
\hline Areia $(\%)$ & 4,0 & 40,0 & 88,0 \\
\hline
\end{tabular}


noso), as doses utilizadas foram equivalentes a $0,3,6,12$ e $24 \mathrm{Mg} \mathrm{ha}^{-1}$. As doses foram determinadas depois da incubação prévia com doses de lodo de curtume variando de 5 a $80 \mathrm{Mg} \mathrm{ha}^{-1}$; o objetivo foi avaliar a quantidade de sódio adicionada pelo resíduo e a elevação do $\mathrm{pH}$ dos solos. De acordo com os resultados da incubação prévia, as doses de $24 \mathrm{Mg} \mathrm{ha}^{-1}$ do NVef (muito argiloso) e o LVAd (argiloso), e de $6 \mathrm{Mg} \mathrm{ha}^{-1}$ do RQo (arenoso) seriam suficientes para elevar o $\mathrm{pH}$ desses solos a 6,0.

Os experimentos foram conduzidos por 105 dias, no escuro e em sala climatizada, com temperatura constante de $28^{\circ} \mathrm{C}$. O delineamento experimental, para cada solo, foi inteiramente casualizado, em fatorial completo 5x21 (cinco doses e 21 épocas de avaliação), com três repetições. O correspondente a cada dose de lodo foi aplicado em $200 \mathrm{~g}$ de solo, que foram acondicionados em potes respirométricos de 1,5 L, com tampa vedante. A umidade do solo foi corrigida para $60 \%$ da capacidade máxima de retenção. Cada pote respirométrico recebeu um frasco contendo solução de $\mathrm{NaOH} 0,5 \mathrm{~mol} \mathrm{~L}^{-1}$, trocada periodicamente conforme as épocas de avaliação: 1, 2, 3, 4, 5, 6, 7, 8, 9, 10, 11, 12, 13, 14, 15, 20, 25, 40, 60, 80 e 105 dias de incubação. A quantificação do carbono liberado na forma de $\mathrm{CO}_{2}$ $\left(\mathrm{C}-\mathrm{CO}_{2}\right)$ foi determinada por meio da titulação do $\mathrm{NaOH}$ contido nos frascos, removido de cada pote respirométrico, com solução padronizada de $\mathrm{HCl}$ 0,5 $\mathrm{mol} \mathrm{L}^{-1}$, usando como indicador a fenolftaleína, e com precipitação prévia do car-

Tabela 2. Caracterização físico-química do lodo de curtume.

\begin{tabular}{|c|c|}
\hline Variável & Concentração \\
\hline $\mathrm{pH}^{(1)}$ & 11,8 \\
\hline $\left.\mathrm{CE}(\mathrm{dS} \mathrm{m})^{-1}\right)^{(1)}$ & 27,6 \\
\hline Umidade a $65^{\circ} \mathrm{C}\left(\mathrm{g} \mathrm{kg}^{-1}\right)$ & 854,8 \\
\hline Carbono total $\left(\mathrm{g} \mathrm{kg}^{-1}\right)$ & 181,8 \\
\hline Carbono orgânico $\left(\mathrm{g} \mathrm{kg}^{-1}\right)$ & 177,0 \\
\hline $\mathrm{N}$ total $\left(\mathrm{g} \mathrm{kg}^{-1}\right)$ & 28,2 \\
\hline $\mathrm{N}$ amoniacal $\left(\mathrm{g} \mathrm{kg}^{-1}\right)$ & 9,6 \\
\hline $\mathrm{N}$ nítrico $\left(\mathrm{g} \mathrm{kg}^{-1}\right)$ & 0,4 \\
\hline Relação C/N & 6,4 \\
\hline $\mathrm{S}$ total $\left(\mathrm{g} \mathrm{kg}^{-1}\right)$ & 12,4 \\
\hline$P$ total $\left(\mathrm{g} \mathrm{kg}^{-1}\right)$ & 3,3 \\
\hline Ca total $\left(\mathrm{g} \mathrm{kg}^{-1}\right)$ & 210,1 \\
\hline Na total $\left(\mathrm{g} \mathrm{kg}^{-1}\right)$ & 59,9 \\
\hline $\operatorname{Mg}$ total $\left(\mathrm{g} \mathrm{kg}^{-1}\right)$ & 2,1 \\
\hline $\mathrm{K}$ total $\left(\mathrm{g} \mathrm{kg}^{-1}\right)$ & 0,6 \\
\hline Mn total $\left(\mathrm{mg} \mathrm{kg}^{-1}\right)$ & 2.307 \\
\hline Fe total $\left(\mathrm{mg} \mathrm{kg}^{-1}\right)$ & 944 \\
\hline $\mathrm{Zn}$ total $\left(\mathrm{mg} \mathrm{kg}^{-1}\right)$ & 48 \\
\hline $\mathrm{Cu}$ total $\left(\mathrm{mg} \mathrm{kg}^{-1}\right)$ & 14 \\
\hline Cr total $\left(\mathrm{mg} \mathrm{kg}^{-1}\right)$ & 798 \\
\hline
\end{tabular}

${ }^{(1)}$ Medidos diretamente no lodo de curtume. bonato mediante a adição de $1 \mathrm{~mL}$ de solução de $\mathrm{BaCl}_{2} 4 \mathrm{~mol} \mathrm{~L}^{-1}$.

Os dados foram interpretados na forma de $\mathrm{C}-\mathrm{CO}_{2}$ acumulado, descontado o valor da testemunha (dose 0 ), até 105 dias de incubação, atribuindo-se essa diferença à mineralização do carbono aplicado por meio do resíduo $\left(\mathrm{C}_{\mathrm{m}}\right)$. Tais valores foram submetidos à análise de variância e regressão não-linear por meio do SAS (SAS Institute, 1999). Todas as análises foram realizadas individualmente para cada solo.

O modelo não linear utilizado foi o de cinética química de primeira ordem: $C_{m}=C_{0}\left(1-e^{-k t}\right)$, em que o $\mathrm{C}_{\mathrm{m}}$ corresponde ao carbono mineralizado no tempo t, em mg $100 \mathrm{~g}^{-1}$; o $\mathrm{C}_{0}$, o carbono potencialmente mineralizável do resíduo, na dose considerada, em mg $100 \mathrm{~g} \mathrm{~g}^{-1}$; k, a constante de velocidade da reação de mineralização do carbono do lodo de curtume, em dias ${ }^{-1} ; \mathrm{e}$ t, o tempo, em dias. A meia-vida de mineralização, correspondente ao tempo necessário para que ocorra a mineralização de metade do carbono potencialmente mineralizável $\left(\mathrm{C}_{0}\right)$ durante os 105 dias de incubação, foi obtida segundo a equação: ln 2/k (Latham, 1974). A fração de mineralização foi calculada no final do período de incubação por meio da equação: $\mathrm{FM}=100 \mathrm{C}_{\mathrm{m}} / \mathrm{C}_{\mathrm{a}}$, em que FM corresponde à fração de mineralização do carbono adicionado por meio da dose do lodo de curtume em porcentagem; $\mathrm{C}_{\mathrm{m}}$, à diferença entre $\mathrm{C}-\mathrm{CO}_{2}$ liberado acumulado no tratamento e na testemunha, em mg $100 \mathrm{~g}^{-1}$; e $\mathrm{C}_{\mathrm{a}}$, à quantidade de carbono adicionado via dose do lodo de curtume, em mg $100 \mathrm{~g}^{-1}$.

\section{Resultados e Discussão}

A quantidade de $\mathrm{C}-\mathrm{CO}_{2}$ mineralizado até 105 dias aumentou em virtude da dose de lodo de curtume aplicada, independentemente da classe textural do solo, sem inibição do processo respiratório microbiano em nenhuma das doses utilizadas (Figura 1). As diferenças entre as quantidades de $\mathrm{C}-\mathrm{CO}_{2}$ liberadas nos tratamentos que receberam doses de lodo e nos que não receberam (testemunhas) indicam a contribuição positiva dos resíduos na atividade microbiana edáfica.

A atividade microbiana foi mais intensa nos primeiros 15 a 20 dias de incubação, com posterior decréscimo (Figura 1). Esse comportamento pode ser atribuído à mineralização inicial do carbono orgânico prontamente oxidável, cuja exaustão conduz à redução do fluxo de C- $\mathrm{CO}_{2}$, como no trabalho de Andrade (2004), em que o autor encontrou valores crescentes de coefi- 
ciente de correlação entre os compostos facilmente degradáveis do lodo de esgoto e a FM do carbono, em decorrência do tempo de incubação.

Segundo Castilhos et al. (2000), a maior liberação de $\mathrm{C}-\mathrm{CO}_{2}$ em solos tratados com lodo de curtume não se deve apenas à incorporação de matéria orgânica e
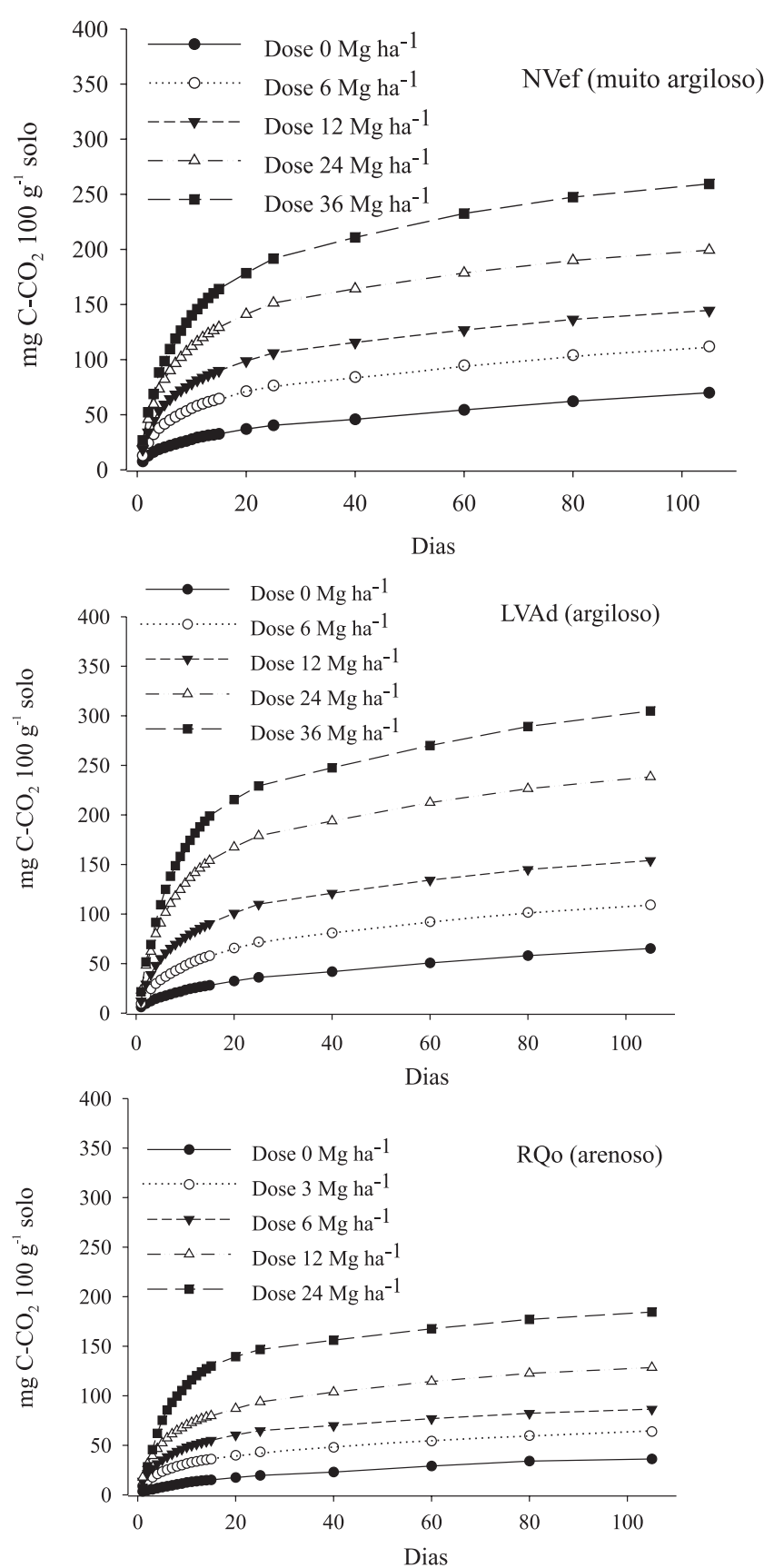

Figura 1. Quantidades de carbono liberado, acumulado por 105 dias, na forma de $\mathrm{CO}_{2}\left(\mathrm{C}-\mathrm{CO}_{2}\right)$, em três solos: NVef, LVAd e RQo. nutrientes, mas também ao efeito corretivo e à ação inoculante do lodo de curtume, que possui microrganismos adaptados ao meio e atuantes na mineralização dos resíduos.

Os valores médios da FM, usando os resultados obtidos nas doses 6, 12 e $24 \mathrm{Mg} \mathrm{ha}^{-1}$ (Tabela 3), comuns aos três solos, foram: 68\% para o NVef (muito argiloso); $80 \%$ para o LVAd (argiloso); e $82 \%$ para o RQo (arenoso). Esses valores estão muito acima de outros encontrados em lodos de curtume, entre 8 e 16\%, em 240 dias de incubação (Konrad \& Castilhos, 2001), e em lodos de esgoto, entre 7 e 25\%, em 70 dias de incubação (Andrade, 2004).

Valores elevados da fração de mineralização podem ser atribuídos principalmente à baixa relação $\mathrm{C} / \mathrm{N}$ e à composição da fração orgânica. Andrade (2004), ao estudar a cinética de mineralização de quatro lodos de esgoto, em dose correspondente a $40 \mathrm{Mg} \mathrm{ha}^{-1} \mathrm{e}$ com relação $\mathrm{C} / \mathrm{N}$ nos resíduos inferiores a 12 , encontrou FM entre 7 e $22 \%$ e valores de meia-vida entre 11 e 33 dias. Essas diferenças podem estar relacionadas com a matéria orgânica recalcitrante, decorrente do processo biológico e de estabilização da carga orgânica, à que foram submetidos na estação de tratamento.

Tabela 3. Média do carbono adicionado via lodo de curtume (mg $100 \mathrm{~g} \mathrm{~g}^{-1}$ ) e liberado como $\mathrm{CO}_{2}$ (mg $100 \mathrm{~g}^{-1}$ ) em 105 dias de incubação, média do carbono mineralizado (mg $100 \mathrm{~g}^{-1}$ ) e da fração de mineralização (FM, \%), segundo os solos estudados ${ }^{(1)}$.

\begin{tabular}{rrrrc}
\hline $\begin{array}{c}\text { Dose de } \\
\text { lodo } \\
\left(\mathrm{Mg} \mathrm{ha}^{-1}\right)\end{array}$ & Adicionado & Liberado $^{(2)}$ & Mineralizado & $\mathrm{FM}^{(2)}$ \\
\cline { 2 - 4 } & \multicolumn{5}{c}{ NVef (muito argiloso) } \\
0 & - & $69,98 \pm 2,58$ & - & - \\
6 & 54,54 & $111,97 \pm 0,35$ & 41,99 & $76,99 \pm 0,64$ \\
12 & 109,08 & $144,53 \pm 0,55$ & 74,55 & $68,34 \pm 0,48$ \\
24 & 218,16 & $199,31 \pm 1,54$ & 129,32 & $59,28 \pm 0,70$ \\
36 & 327,24 & $259,34 \pm 1,46$ & 189,36 & $57,87 \pm 0,14$ \\
\hline \multicolumn{5}{c}{ LVAd (argiloso) } \\
0 & - & $65,21 \pm 0,50$ & - & - \\
6 & 54,54 & $109,20 \pm 0,22$ & 43,99 & $80,66 \pm 0,40$ \\
12 & 109,08 & $153,99 \pm 1,56$ & 88,78 & $81,39 \pm 1,43$ \\
24 & 218,16 & $238,41 \pm 0,70$ & 173,20 & $79,39 \pm 0,32$ \\
36 & 327,24 & $304,93 \pm 0,51$ & 239,72 & $73,26 \pm 0,16$ \\
\hline \multicolumn{5}{c}{ RQo (arenoso) } \\
0 & - & $36,20 \pm 0,06$ & - & - \\
3 & 27,27 & $63,82 \pm 1,34$ & 27,62 & $101,28 \pm 4,89$ \\
6 & 54,54 & $86,40 \pm 1,98$ & 50,20 & $92,04 \pm 3,46$ \\
12 & 109,08 & $128,47 \pm 0,49$ & 92,27 & $84,59 \pm 0,45$ \\
24 & 218,16 & $184,48 \pm 0,92$ & 148,28 & $67,97 \pm 0,42$ \\
\hline
\end{tabular}

${ }^{(1)} \mathrm{FM}(\%)=100 \mathrm{C}_{\mathrm{m}} / \mathrm{C}_{\mathrm{a}}$. (2)Média \pm desvio-padrão. 
O cromo na forma trivalente $\left(\mathrm{Cr}^{3+}\right)$, presente no lodo de curtume, parece não ter influenciado a mineralização do carbono aplicado por meio do resíduo, provavelmente pelo fato de encontrar-se em baixa concentração, quando comparado a outros lodos de curtume, que não separam os efluentes que contêm cromo, nos quais podem ser encontrados até $36.000 \mathrm{mg} \mathrm{kg}^{-1}$ (Konrad \& Castilhos, 2002). Ou ainda, pela possibilidade do $\mathrm{Cr}^{3+}$ encontrar-se complexado com a matéria orgânica do resíduo, mesmo quando em altas concentrações (Aquino Neto \& Camargo, 2000). Resultados semelhantes foram observados por outros autores (Castilhos et al., 2000; Ferreira et al., 2003).

Jahnel et al. (1999) relataram que a aplicação de $50 \mathrm{mg} \mathrm{kg}^{-1}$ de $\mathrm{Cr}^{6+}$, na forma de $\mathrm{K}_{2} \mathrm{Cr}_{2} \mathrm{O}_{7}$, a uma amostra de solo argiloso, reduziu o número total de bactérias no solo em relação à testemunha, enquanto essa mesma dose de $\mathrm{Cr}^{6+}$, quando adicionada na forma de bagaço de cana $\left(10 \mathrm{~g} \mathrm{~kg}^{-1}\right)$ e composto orgânico (30 $\left.\mathrm{g} \mathrm{kg}^{-1}\right)$, não proporcionou tal redução. Assim, verificou-se que o cromo complexado com a matéria orgânica na solução do solo não provoca alteração no número de bactérias.

Barajas-Aceves \& Dendooven (2001), ao estudar a mineralização do carbono em diferentes solos que receberam $12 \mathrm{Mg} \mathrm{ha}^{-1}$ (base seca) de lodo de curtume com cromo (6.690 $\mathrm{mg} \mathrm{kg}^{-1}$ ), obtiveram valor médio da FM igual a 31\%, em 70 dias de incubação. Segundo esses autores, a fração de mineralização foi diferente em cada solo estudado, sugerindo que essa diferença pode ser causada pela diversidade na atividade microbiana de cada solo. Neste trabalho, diferenças também foram verificadas entre os solos.

As testemunhas dos solos NVef (muito argiloso) e LVAd (argiloso) apresentaram valores mais elevados de $\mathrm{C}-\mathrm{CO}_{2}$, comparativamente à testemunha do solo RQo (arenoso), o que foi indicativo de maior atividade microbiana dos solos argilosos sob condições originais. Contudo, não se observou relação evidente entre FM do carbono do lodo de curtume e a atividade microbiana original dos solos, devendo a diversidade microbiana ser fator fundamental na magnitude dessa mineralização, tal qual sugerido por Barajas-Aceves \& Dendooven (2001).

Nos três solos estudados, o valor da FM diminuiu com o aumento da dose, o que pode estar relacionado ao aumento do conteúdo orgânico adicionado, suplantando a capacidade de mineralização dos microrganismos do solo (Wong et al., 1998). Contudo, quanto ao RQo (arenoso), a adição de $6 \mathrm{Mg} \mathrm{ha}^{-1}$ gerou uma FM média superior a $100 \%$ (Tabela 3), indicando que, nesse solo e dose, o lodo de curtume pode ter provocado estímulo aos microrganismos decompositores, os quais, além de consumir todo o carbono adicionado por meio do lodo, também podem ter degradado a matéria orgânica nativa do solo, o que se denomina positive priming effect ou efeito "priming” positivo. Segundo revisão realizada por Kuzyakov et al. (2000), as causas, mecanismos e fontes que geram esse efeito ainda não estão elucidadas. No entanto, os autores citam que a adição de N, principalmente na forma amoniacal, bem como de substâncias orgânicas facilmente biodegradáveis, acelera a mineralização da matéria orgânica do solo (MOS), em função da redução da relação C/N da MOS ou por cometabolismo, respectivamente.

O lodo utilizado neste trabalho teria o potencial de promover o chamado efeito priming positivo, uma vez que o material orgânico do lodo é relativamente de fácil decomposição, o que se confirma por meio da alta fração de mineralização (Tabela 3), além de apresentar expressiva quantidade do total de $\mathrm{N}$ do resíduo sob forma amoniacal, cerca de 35\% (Tabela 2).

Com relação à cinética de mineralização, os dados de $\mathrm{C}-\mathrm{CO}_{2}$ se ajustaram ao modelo de primeira ordem (Tabela 4). Os valores de $\mathrm{C}_{\mathrm{o}}$ aumentaram com o carbono aplicado por meio de doses de lodo em todos os solos, ao passo que os valores da constante de velocidade de mineralização $(\mathrm{k})$ tenderam a diminuir com a dose nos solos de textura muito argilosa e arenosa; não foi observada tendência no LVAd (argiloso) (Tabela 4).

Em virtude dos elevados valores de k, a meia-vida foi baixa, em média igual a seis dias, com valores variando

Tabela 4. Parâmetros de cinética química e meia-vida de mineralização obtidos a partir dos ajustes dos dados de carbono liberado a equações de cinética química de primeira or$\operatorname{dem}^{(1)}$.

\begin{tabular}{ccccc}
\hline $\begin{array}{c}\text { Dose de lodo } \\
\left(\mathrm{Mg} \mathrm{ha}^{-1}\right)\end{array}$ & $\begin{array}{c}\mathrm{C}_{\mathrm{o}} \\
\left(\mathrm{mg} \mathrm{100g}^{-1}\right)\end{array}$ & $\begin{array}{c}\mathrm{k} \\
\left(\mathrm{dia}^{-1}\right)\end{array}$ & $\begin{array}{c}\text { Meia-vida } \\
(\mathrm{dia})\end{array}$ & $\mathrm{R}^{2}$ \\
\hline 6 & 38,97 & 0,1365 & 5 & $0,99^{* *}$ \\
12 & 69,70 & 0,1403 & 5 & $0,99^{* *}$ \\
24 & 121,00 & 0,1242 & 6 & $0,99^{* *}$ \\
36 & 175,40 & 0,1030 & 7 & $0,99^{* *}$ \\
\hline \multicolumn{5}{c}{ LVAd (argiloso) } \\
6 & 41,58 & 0,0924 & 8 & $0,99^{* *}$ \\
12 & 82,68 & 0,1072 & 6 & $0,99^{* *}$ \\
24 & 160,40 & 0,1128 & 6 & $0,99^{* *}$ \\
36 & 221,90 & 0,1022 & 7 & $0,99^{* *}$ \\
\hline \multicolumn{5}{c}{ RQo (arenoso) } \\
3 & 24,21 & 0,1827 & 4 & $0,99^{* *}$ \\
6 & 46,18 & 0,1659 & 4 & $0,99^{* *}$ \\
12 & 82,13 & 0,1311 & 5 & $0,99^{* *}$ \\
24 & 139,40 & 0,1214 & 6 & $0,99^{* *}$ \\
\hline
\end{tabular}

${ }^{(1)} \mathrm{C}_{\mathrm{m}}=\mathrm{C}_{\mathrm{o}}\left(1-\mathrm{e}^{-\mathrm{kt}}\right) ; \mathrm{R}^{2}=$ coeficiente de determinação. ${ }^{* *}$ Significativo a $1 \%$ de probabilidade. 
de quatro a oito (Tabela 4). Isto significa que, ao final dos dez primeiros dias de incubação, mais da metade do $\mathrm{C}_{\mathrm{o}}$ foi liberado na forma de $\mathrm{C}-\mathrm{CO}_{2}$, evidenciando, assim, uma fase de rápida mineralização, seguida de outra mais lenta e de estabilização dos fluxos de $\mathrm{C}-\mathrm{CO}_{2}$. Esse comportamento é comum quando se adicionam resíduos orgânicos a solos agrícolas (Sommers et al., 1979; Bernal et al., 1998).

\section{Conclusões}

1. A atividade microbiana aumenta com a aplicação de doses crescentes de lodo de curtume, independentemente da classe textural do solo.

2. A fração de mineralização do carbono adicionado por meio do lodo de curtume é alterada de acordo com a classe textural.

3. O processo de mineralização do carbono adicionado por meio do lodo de curtume é dividido em duas fases distintas; uma de aproximadamente seis dias, com mineralização intensa, e outra mais lenta, na seqüência, observando-se a tendência de redução e estabilização do fluxo de carbono na forma de $\mathrm{CO}_{2}$.

\section{Agradecimentos}

Ao CNPq, pelas bolsas de mestrado e de produtividade concedidas, respectivamente, a Alexandre Martin Martines e a Elke Jurandy Bran Nogueira Cardoso; ao Curtume Vanzella Ltda., pelo apoio técnico e concessão do resíduo; a Denise de Lourdes Colombo Mescolotti e Luis Fernando Baldesin, pela colaboração na realização dos experimentos.

\section{Referências}

ABREU, M.F.; ABREU, C.A.; ANDRADE, J.C. Determinação de fósforo, potássio, cálcio, magnésio, enxofre, cobre, ferro, manganês, zinco, níquel, cádmio, cromo e chumbo em ácido nítrico usando métodos da US-EPA. In: RAIJ, B. van; ANDRADE, J.C.; CANTARELLA, H.; QUAGGIO, J.A. (Ed.). Análise química para avaliação da fertilidade de solos tropicais. Campinas: Instituto Agronômico, 2001. p.251-261.

ANDRADE, C.A. Fração orgânica de biossólidos e efeito no estoque de carbono e qualidade da matéria orgânica de um latossolo cultivado com eucalipto. 2004. 121p. Tese (Doutorado) - Escola Superior de Agricultura Luiz de Queiroz, Piracicaba.

AQUINO NETO, V.; CAMARGO, O.A. Crescimento e acúmulo de crômio em alface cultivada em dois latossolos tratados com $\mathrm{CrCl}_{3}$ e resíduos de curtume. Revista Brasileira de Ciência do Solo, v.24, p.225-235, 2000.
BARAJAS-ACEVES, M.; DENDOOVEN, L. Nitrogen, carbon and phosphorus mineralization in soils from semi-arid highlands of central Mexico amended with tannery sludge. Bioresource Technology, v.77, p.121-130, 2001.

BERNAL, M.P.; SÁNCHEZ-MONEDERO, M.A.; PAREDES, C.; ROIG, A. Carbon mineralization from organic wastes at different composting stages during their incubation with soil. Agriculture, Ecosystems and Environment, v.69, p.175-189, 1998.

BROOKES, P.C. The use of microbial parameters in monitoring soil pollution by heavy metals. Biology and Fertility of Soils, v.19, p.269-279, 1995.

CAMARGO, O.A.; MONIZ, A.C.; JORGE, J.A.; VALADARES, J.M.A.S. Métodos de análise química, mineralógica e física de solos do Instituto Agronômico de Campinas. Campinas: Instituto Agronômico, 1986. 94p. (Boletim técnico, 106).

CASTILHOS, D.D.; VIDOR, C.; CASTILHOS, R.M.V. Atividade microbiana em solo suprido com lodo de curtume e cromo hexavalente. Revista Brasileira de Agrociência, v.6, p.71-76, 2000.

CLAAS, I.C.; MAIA, R.A.M. Manual básico de resíduos industriais de curtume. Porto Alegre: Senai, 1994. 664p.

DOMSCH, K.H.; JAGNOW, G.; ANDERSON, T.H. An ecological concept for the assessment of side-effects of agrochemicals on soil microorganisms. Residue Reviews, v.86, p.65-105, 1983.

FERREIRA, A.S.; CAMARGO, F.A.O.; TEDESCO, M.J.; BISSANI, C.A. Alterações de atributos químicos e biológicos de solo e rendimento de milho e soja pela utilização de resíduos de curtume e carbonífero. Revista Brasileira de Ciência do Solo, v.27, p.755-763, 2003.

FIGLIOLIA, A.; BENEDETTI, A.; DELL’ABATE, M.T.; IZZA, C.; INDIATI, R. Potential chromium bio-availability by Lactuca sativa grown on two soils amended with tannery leather residues. Fresenius Environmental Bulletin, v.1, p.406-410, 1992.

IBGE. Produção da pecuária municipal 2003. Rio de Janeiro, 2003. v.31, 31p.

JAHNEL, M.C.; CARDOSO, E.J.B.N.; DIAS, C.T.S. Determinação do número mais provável de microrganismos do solo pelo método de plaqueamento por gotas. Revista Brasileira de Ciência do Solo, v.23, p.553-559, 1999.

KIEHL, E.J. Fertilizantes orgânicos. São Paulo: Agronômica Ceres, 1985. 492p.

KONRAD, E.E.; CASTILHOS, D.D. Alterações químicas do solo e crescimento do milho decorrente da adição do lodo de curtume. Revista Brasileira de Ciência do Solo, v.26, p.257-265, 2002.

KONRAD, E.E.; CASTILHOS, D.D. Atividade microbiana em um planossolo após a adição de resíduos de curtume. Revista Brasileira de Agrociência, v.7, p.131-135, 2001.

KUZYAKOV, Y.; FRIEDEL, J.K.; STAHR, K. Review of mechanisms and quantification of priming effects. Soil Biology and Biochemistry, v.32, p.1485-1498, 2000.

LATHAM, J.L. Cinética elementar de reação. São Paulo: E. Blücher, 1974. 112p. 
LUZ, C. Mercado de couro curte crescimento tanto interno quanto externo. Latin Chemical, v.9, p.35-37, 2003.

RAIJ, B. van; ANDRADE, J.C.; CANTARELLA, H.; QUAGGIO, J.A. (Ed.). Análise química para avaliação da fertilidade de solos tropicais. Campinas: Instituto Agronômico, 2001. 285p.

SAS INSTITUTE (Cary, Estados Unidos). SAS/STAT: user's guide. Version 8.02. Cary, 1999. 3v.

SELBACH, P.A.; TEDESCO, M.J.; GIANELLO, C.; CAVALLET, L.E. Descarte e biodegradação de lodos de curtume no solo. Revista do Couro, v.4, p.51-62, 1991.
SILVA, F.C. da (Org.). Manual de análises químicas de solos, plantas e fertilizantes. Brasília: Embrapa-SCT, 1999. 370p.

SOMMERS, L.E.; NELSON, D.W.; SILVIERA, D.J. Transformations of carbon, nitrogen, and metals in soils treated with waste materials. Journal of Environmental Quality, v.8, p.287294, 1979.

WONG, J.W.C.; LAI, K.M.; FANG, M.; MA, K.K. Effect of sewage sludge amendment on soil microbial activity and nutrient mineralization. Environment International, v.24, p.935-943, 1998.

Recebido em 3 de junho de 2005 e aprovado em 3 de março de 2006 